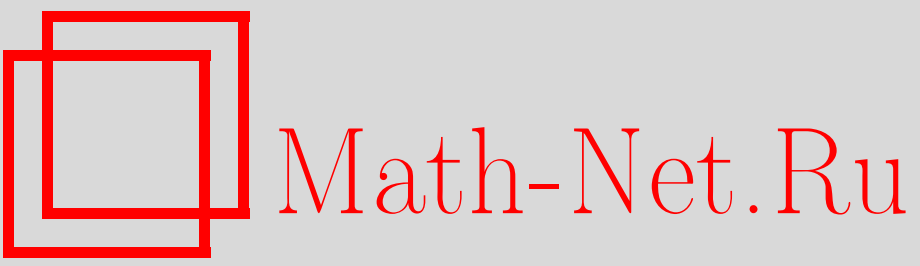

В. П. Цветков, Интегральное уравнение для спинорной амплитуды дираковской частицы в искривленном пространстве-времени, ТМФ, 2003, том 135, номер 2, 331-337

DOI: https://doi.org/10.4213/tmf191

Использование Общероссийского математического портала Math-Net.Ru подразумевает, что вы прочитали и согласны с пользовательским соглашением

http: //www . mathnet.ru/rus/agreement

Параметры загрузки:

IP : 35.173 .219 .149

26 апреля 2023 г., 12:41:59 


\section{ИНТЕГРАЛЬНОЕ УРАВНЕНИЕ ДЛЯ СПИНОРНОЙ АМПЛИТУДЫ ДИРАКОВСКОЙ ЧАСТИЦЫ В ИСКРИВЛЕННОМ ПРОСТРАНСТВЕ-ВРЕМЕНИ}

Получена система интегральных уравнений для спинорной амплитуды волнового пакета, описывающей массивную нейтральную дираковскую частицу в искривленном пространстве-времени произвольной геометрии. Это уравнение позволяет описать спиновую динамику фермионов в гравитационных полях адекватно квантовой природе спина. Рассмотрен конкретный пример метрики Керра-Шилда. Обсуждается вопрос об осцилляции массивных нейтрино во внешнем гравитационном поле.

Ключевые слова: интегральное уравнение, спинорная амплитуда, искривленное пространство-время.

В связи с наличием астрофизических компактных объектов, вблизи которых существуют сильные гравитационные поля, несомненный интерес представляет изучение массивных нейтральных дираковских частиц в искривленном пространстве-времени произвольной геометрии. Это прежде всего связано с изучением нейтринных процессов, играющих важную роль в эволюции компактных астрофизических объектов (нейтронных звезд, черных дыр).

Наблюдаемый дефицит солнечных нейтрино потребовал пересмотреть представления о характере термоядерных явлений, происходящих на солнце, и физике нейтринных процессов.

На ускорителях и в астрофизических условиях генерируются только левые нейтрино $\nu_{\mathrm{L}}$ и правые антинейтрино $\bar{\nu}_{\mathrm{R}}$, спин которых направлен против и по направлению импульса частицы, соответственно. Только они взаимодействуют с вешеством, и, следовательно, наблюдаются. Нейтрино $\nu_{\mathrm{R}}$ и $\bar{\nu}_{\mathrm{L}}$ с вешеством практически не взаимодействуют, и оно для них прозрачно. Если у нейтрино нет массы, то их спиральность не меняется даже при наличии внешнего гравитационного поля. Но если они массивны $\left(m_{\nu} \neq 0\right.$, хотя и очень мало), то возможны нейтринные осцилляции - переходы $\nu_{\mathrm{L}} \leftrightarrow \nu_{\mathrm{R}}$ и $\bar{\nu}_{\mathrm{R}} \leftrightarrow \bar{\nu}_{\mathrm{L}}-$ в гравитационных полях. Актуальность данной тематики резко возрастает после сообщений ряда экспериментальных групп [1], [2] о наличии у нейтрино ненулевой массы покоя $2 \cdot 10^{-2}{ }_{\ni} \mathrm{B}<m_{\nu}<7 \cdot 10^{-2}{ }_{\ni} \mathrm{B}$.

\footnotetext{
* Тверской государственный университет, Тверь, Россия. E-mail: tsvet@tversu.ru
} 
Спиновая динамика нейтральной массивной дираковской частицы в искривленном пространстве-времени рассматривалась в ряде работ [3]-[6].

В данной статье, являющейся непосредственным продолжением работ [3]-[5], сформулирован принципиально новый математический подход к изучению эволюции спинорной амплитуды дираковской частицы в искривленном пространстве-времени, в основе которого лежит система интегральных уравнений с вольтеровскими ядрами.

Движение массивной нейтральной дираковской частицы со спином $1 / 2$ будем описывать общековариантным уравнением Дирака

$$
i\left[e_{(a)}^{\mu} \gamma^{a} \partial_{\mu}-\frac{1}{4} \gamma_{a b c} \gamma^{c} \gamma^{b} \gamma^{a}\right] \Psi=m \Psi
$$

где $\gamma_{a b c}=e_{(a) \nu ; \mu} e_{(b)}^{\nu} e_{(c)}^{\mu}-$ коэффишиенты врашения Риччи, $\gamma^{a}$ - матрищы Дирака, $m-$ масса частицы, $c=\hbar=1$.

Тетрадные векторы предполагаются ортогональными:

$$
g_{\mu \nu} e_{(a)}^{\mu} e_{(b)}^{\nu}=\eta_{a b}
$$

где $g_{\mu \nu}$ - метрический тензор, $\eta_{a b}$ - матрица Минковского.

Как и в [5], будем искать решение общековариантного уравнения Дирака, имеющее вид распространяющегося с течением времени волнового пакета, локализованного в пространственной области шириной $d^{-1}$ :

$$
\Psi=\frac{d^{-3 / 2}}{2 \sqrt{2} \pi^{9 / 4}} \int d^{3} q \exp \left\{-\left[i \int_{t}^{\infty} E(\vec{q}, \tau) d \tau+i(\vec{q} \vec{r})+\frac{\left(\vec{q}-\vec{q}_{0}\right)}{2 d^{2}}\right]\right\} U(\vec{q}, t) .
$$

Будем считать выполненным естественное условие $\kappa=d^{-1} / l_{\mathrm{g}} \ll 1$, где $l_{\mathrm{g}}$ - характерный масштаб изменения метрики. Очевидно, что $l_{\mathrm{g}}$ имеет тот же порядок, что и размеры источника гравитационного поля.

Волновая функция $\Psi$ нормируется согласно условию

$$
\int \bar{\Psi} e_{(a)}^{0} \gamma^{a} \Psi \sqrt{-g} d^{3} x=1
$$

Тогда из $(2),(3)$ следует асимптотическое условие нормировки для $U(\vec{q}, t)$ :

$$
U^{+}(\vec{q}, t \rightarrow \infty) U(\vec{q}, t \rightarrow \infty)=1
$$

Для описания спиновой динамики дираковской частицы представим $\Psi$ в виде разложения по спиральным состояниям

$$
\Psi=\Psi_{\mathrm{L}}+\Psi_{\mathrm{R}}
$$

где

$$
\Psi_{\mathrm{L}, \mathrm{R}}=\frac{1}{2}\left(1 \pm \gamma^{5}\right) \Psi, \quad \gamma^{5}=\left(\begin{array}{cc}
0 & I \\
-I & 0
\end{array}\right)
$$


$I$ - единичная $(2 \times 2)$-матрица.

Отсюда следуют выражения для вероятностей $W_{\mathrm{L}}$ и $W_{\mathrm{R}}$ соответственно левого и правого состояний массивной дираковской частицы:

$$
W_{\mathrm{L}, \mathrm{R}}=\iiint \bar{\Psi}_{\mathrm{L}, \mathrm{R}} e_{(a)}^{0} \gamma^{a} \Psi_{\mathrm{L}, \mathrm{R}} \sqrt{-g} d^{3} x
$$

Вид функции $E(\vec{q}, \tau)$ будет уточнен в дальнейшем. Очевидно, что $E(\vec{q}, \tau)$ удовлетворяет асимптотическому условию $E(\vec{q}, \tau \rightarrow \infty) \rightarrow \sqrt{q^{2}+m^{2}}$.

Разлагая компоненты метрического тензора, а следовательно, и коэффициенты обшековариантного уравнения Дирака по степеням малого параметра $\kappa$ в окрестности центра локализации волнового пакета, решение этого уравнения можно свести к решению системы линейных обыкновенных дифференциальных уравнений, описьваюшей эволюцию спинорной амплитуды дираковской частицы $U(\vec{q}, t)(\vec{q}-$ импульс частицы $)$.

Основной вклад в волновой пакет дают значения $\vec{q}$ вблизи $\vec{q}_{0}$ в интервале шшириной $d^{-1}$. Для исследования спиновой динамики представим $U$ в виде линейной комбинации полей с правой $\left(U_{\mathrm{R}}\right)$ и левой $\left(U_{\mathrm{L}}\right)$ спиральностями: $U=U_{\mathrm{L}}+U_{\mathrm{R}}$, где $U_{\mathrm{L}, \mathrm{R}}=\left(1 \pm \gamma_{5}\right) U / 2$

Четырехкомпонентные спиноры $U_{\mathrm{L}}$ и $U_{\mathrm{R}}$ можно выразить через двухкомпонентные спиноры $F$ и $H$ следуюшим образом:

$$
U_{\mathrm{L}}=\left(\begin{array}{c}
F \\
-F,
\end{array}\right), \quad U_{\mathrm{R}}=\left(\begin{array}{c}
H \\
H
\end{array}\right)
$$

Из $F$ и $H$ составим четырехкомпонентный спинор

$$
R=\left(\begin{array}{c}
F \\
H
\end{array}\right) .
$$

Этот спинор удовлетворяет уравнению, которое можно представить в форме уравнения Шредингера

$$
\dot{R}=-i \widehat{H} R \text {. }
$$

Точное значение $\widehat{H}$ в случае метрики Керра-Шилда приводится в [5], а в случае слабоискривленного пространства-времени произвольной геометрии в первом постньютоновском приближении дано в [6].

Обозначим $\widehat{H}(t \rightarrow \infty)=\widehat{H}_{0}$, решение $R(t \rightarrow \infty)=R_{0}$, импульс дираковской частицы $-\vec{q}$, единичный вектор в направлении импульса $-\vec{n}=\vec{q} / q(q=|\vec{q}|)$. Введем в рассмотрение двухрядные матрицы $\sigma_{ \pm}=(1 \pm(\vec{\sigma} \vec{n}))$ ( $\vec{\sigma}$ - матрицы Паули). Из них составим четырехрядные матрицы

$$
\widehat{S}^{ \pm}=\frac{1}{2\left(\kappa_{01}+1\right)}\left(\begin{array}{cc}
\sigma_{ \pm}+\kappa_{01} & \pm \kappa_{0} \\
\pm \kappa_{0} & \sigma_{\mp}+\kappa_{01}
\end{array}\right),
$$

где $\kappa_{0}=m / q, \kappa_{01}=\sqrt{1+\kappa_{01}^{2}}-1$. 
Тогда

$$
\widehat{H}_{0}=q \widehat{S}^{+}, \quad R_{0}=\widehat{S}^{-}\left(\begin{array}{c}
r_{01} \\
r_{02}
\end{array}\right),
$$

где $r_{01}, r_{02}$ - собственные векторы матришы $(\vec{\sigma} \vec{n})$ :

$$
(\vec{\sigma} \vec{n}) r_{01,02}=\mp r_{01,02} .
$$

Отметим свойство ортогональности $\widehat{S}^{+}$и $\widehat{S}^{-}: \widehat{S}^{+} \widehat{S}^{-}=0$, которое проверяется непосредственно и в дальнейшем будет играть важную роль. Разность $\widehat{H}-\widehat{H}_{0}$ обозначим $\widehat{V}$.

После простых преобразований систему дифференциальных уравнений (9) можно представить в виде системы интегральных уравнений с вольтерровыми ядрами:

$$
R=i \int_{t}^{\infty}\left(-\widehat{V}(\tau)+{ }^{+} \widehat{V}(\tau) e^{2 i \alpha(\tau-\mathrm{t})}\right) R(\tau) d \tau+R_{0},
$$

где $\alpha=\sqrt{q^{2}+m^{2}}, \mp \widehat{V}=\widehat{S} \mp \widehat{V}$.

Решение (12) может быть построено методом последовательных приближений:

$$
R^{(n)}=i \int_{t}^{\infty}\left({ }^{-} \widehat{V}(\tau)+{ }^{+} \widehat{V}(\tau) e^{2 i \alpha(\tau-\mathrm{t})}\right) R^{(n-1)}(\tau) d \tau+R_{0} .
$$

В качестве начального приближения выбирается $R^{(0)}=R_{0}$. В пределе $R=$ $\lim _{n \rightarrow \infty} R^{(n)}$ будет являться точным решением уравнения (12).

Ядра системы интегральных уравнений содержат члены с быстроосциллируюшим множителем $\mathrm{e}^{2 i \alpha(\tau-\mathrm{t})}$. Отметим, что для сходимости данных интегралов достаточным является условие $+\widehat{V}(\tau \rightarrow \infty) \rightarrow 0$.

Для сходимости интегралов с членами $-\widehat{V}(\tau)$ необходимо выполнение более сильного условия:

$$
\left.\left.\right|^{-} \widehat{\mathrm{V}}_{i k}\right|_{i, k=0,1,2,3}<\frac{A}{\left(r_{0}+\tau\right)^{1+\delta}},
$$

где $\delta>0, r_{0}$ - характерный радиус гравитируюшего объекта.

Основной вклад в интегралы, содержашие множитель $e^{2 i \alpha(\tau-t)}$, будет давать область интегрирования $t-\Delta \tau \leqslant \tau \leqslant t+\Delta \tau, \Delta \tau \sim 1 / \alpha$. Если $1 /\left(\alpha l_{\mathrm{g}}\right) \ll 1$, что выполняется всегда, интегралы с $\mathrm{e}^{i \alpha(\tau-\mathrm{t})}$ могут быть оценены на основании следуюшего асимптотического разложения:

$$
\begin{aligned}
J(t) & =\int_{t}^{\infty} f(\tau) e^{i \alpha(\tau-t)} d \tau= \\
& =\frac{i}{\alpha} \sum_{k=0}^{N-1}\left(\frac{i}{\alpha}\right)^{k} \frac{d^{k} f(t)}{d t^{k}}+i\left(\frac{i}{\alpha}\right)^{N} \int_{t}^{\infty}\left(e^{i \alpha(\tau-t)} \frac{d^{N} f(\tau)}{d t^{N}}\right) d \tau .
\end{aligned}
$$

Если $f(t)$ удовлетворяет условию

$$
\left|\frac{d^{N} f(t)}{d t^{N}}\right|<\frac{\alpha A(N-1) !}{\left(t_{0}+t\right)^{N}}
$$


при $N>1$, то

$$
J(t)=\frac{i}{\alpha} \sum_{k=0}^{N-1}\left(\frac{i}{\alpha}\right)^{k} \frac{d^{k} f(t)}{d t^{k}}+O\left(\frac{(N-2) !}{\alpha\left(t_{0}+t\right)^{N-1}}\right) .
$$

В нашем случае $\alpha t_{0}=\alpha l_{\mathrm{g}} \gg 1$, и отбрасываемые члены будут малы до значений $N \sim \alpha t_{0}$. Выбор $N$ будет определяться конкретным порядком приближения в (17). Так, для $R^{(1)}$ достаточно взять $N=1$, для $R^{(2)}-N=2$ и т.д. $\left(N=n\right.$ для $\left.R^{(n)}\right)$. Данная процедура позволяет представить $R$ в виде асимптотического ряда по степеням малого параметра $1 /\left(\alpha l_{\mathrm{g}}\right)$.

Остановимся на выборе функции $E(\vec{q}, \tau)$. В каждом приближении она выбирается из условия сокрашения в $R^{(n)}$ всех больших членов порядка $\left(q l_{\mathrm{g}}\right)$. Это соответствует очевидному физическому условию отсутствия в спинорной амплитуде быстроменяющихся со временем членов.

Сходимость последовательности $R^{(n)}$ доказывается аналогично соответствуюшему доказательству для уравнения Вольтерра второго рода.

Наибольший физический интерес в данном исследовании представляют переходы с изменением спиральности массивной дираковской частицы (в астрофизике- нейтрино). Из (12) и (13) следует, что такие переходы возможны, если $\mp \widehat{V}_{12}$ и $\widehat{V}_{21}$ отличны от нуля. Здесь мы представили четырехрядные матришы $\widehat{V}$ в виде двухрядных $\mp \widehat{V}_{a b}(a, b=1,2)$ :

$$
\mp \widehat{V}=\left(\begin{array}{ll}
\mp \widehat{V}_{11} & \mp \widehat{V}_{12} \\
\mp \widehat{V}_{21} & \mp \widehat{V}_{22}
\end{array}\right)
$$

причем для любой геометрии пространства-времени $\mp \widehat{V}_{12}$ и $\mp \widehat{V}_{21}$ отличны от нуля лишш при $m \neq 0$. Отсюда следует, что эффект нейтринных осцилляций $\nu_{\mathrm{L}} \leftrightarrow \nu_{\mathrm{R}}, \bar{\nu}_{\mathrm{L}} \leftrightarrow \bar{\nu}_{\mathrm{R}}$ возможен только для массивных нейтрино. Величина эффекта определяется конкретной геометрией гравитационного поля.

Разработанный нами метод исследования спиновой динамики массивной дираковской частицы предполагается использовать для подробного изучения этого явления в случае конкретной геометрии искривленного пространства-времени. Особый интерес представляет случай слабого гравитационного поля, учитываемого в первом постньютоновском приближении. В этом случае достаточно вычислить несколько первых членов $R^{(n)}$ (до появления неисчезающих эффектов).

Дальнейшее изложение будем проводить в рамках конкретной и наиболее простой геометрии искривленного пространства-времени метрики Керра-Шилда. Компоненты тетрадных векторов $e_{(a)}^{\mu}$ выражаются через компоненты изотропного вектора $\xi^{\mu}$ следующим образом:

$$
e_{(a)}^{\mu}=\delta_{a}^{\mu}+\frac{1}{2} \xi^{\mu} \xi_{a}, \quad \xi_{a}=\eta_{a b} \xi^{b}, \quad \xi^{a} \xi^{b} \eta_{a b}=0
$$

Используя полученное в работе [5] значение $\widehat{H}$ для метрики Керра-Шилда, находим в нашем случае

$$
\widehat{V}=\left(\begin{array}{lr}
E^{\prime}+\left(p_{n}-u_{n}\right) \hat{n}+\left(p_{1}+u_{1}\right) \hat{e}_{1}+\left(p_{2}+u_{2}\right) \hat{e}_{2} & -m\left(\phi+\lambda \xi_{\|} \hat{n}+\lambda \xi_{\perp} \hat{e}_{1}\right) \\
-m\left(\phi-\lambda \xi_{\|} \hat{n}-\lambda \xi_{\perp} \hat{e}_{1}\right) & E^{\prime}-\left(p_{n}+u_{n}\right) \hat{n}-\left(p_{1}-u_{1}\right) \hat{e}_{1}-\left(p_{2}-u_{2}\right) \hat{e}_{2}
\end{array}\right),
$$


где $\vec{n}=\vec{q} / q, \vec{\xi}=\xi_{\|} \vec{n}+\xi_{\perp} \vec{e}_{1}, \vec{e}_{2}=\left[\vec{n} \times \vec{e}_{1}\right], \hat{n}=(\vec{\sigma} \vec{n}), \hat{e}_{1}=\left(\vec{\sigma} \vec{e}_{1}\right), \hat{e}_{2}=\left(\vec{\sigma} \vec{e}_{2}\right), \vec{v}=v_{\|} \vec{n}+$ $v_{1} \vec{e}_{1}+v_{2} \vec{e}_{2}, \vec{v}=\xi \partial \vec{\xi} / \partial t+(\vec{\xi} \vec{\nabla}) \vec{\xi}, \xi=\xi^{0}, \vec{\xi}=\left(\xi^{1}, \xi^{2}, \xi^{3}\right)$,

$$
\begin{gathered}
E^{\prime}=E-\frac{\xi \xi_{\|}}{1+\xi^{2}} q-i \frac{\partial \xi^{2} / \partial t+\vec{\nabla}(\xi \vec{\xi})}{4\left(1+\xi^{2}\right)}, \\
p_{n}=\frac{-\xi^{2}+\xi_{\perp}^{2} / 2}{1+\xi^{2}} q+\frac{i \xi \xi_{\|}}{4\left(1+\xi^{2}\right)}\left[\frac{\xi}{2}(\vec{\nabla} \xi \cdot \vec{\xi})-(\vec{\nabla} \vec{\xi})-\left(1-\frac{\xi^{2}}{2}\right) \frac{\partial \xi}{\partial t}\right]-i \frac{1+\xi^{2} / 2}{4\left(1+\xi^{2}\right)} v_{\|}, \\
p_{1}=-\frac{\xi_{\|} \xi_{\perp}}{2\left(1+\xi^{2}\right)} q+\frac{i \xi \xi_{\perp}}{4\left(1+\xi^{2}\right)}\left[\frac{\xi}{2}(\vec{\nabla} \xi \cdot \vec{\xi})-(\vec{\nabla} \vec{\xi})-\left(1-\frac{\xi^{2}}{2}\right) \frac{\partial \xi}{\partial t}\right]-i \frac{1+\xi^{2} / 2}{4\left(1+\xi^{2}\right)} v_{1}, \\
p_{2}=-i \cdot \frac{1+\xi_{\perp}^{2} / 2}{4\left(1+\xi^{2}\right)} v_{2}, \quad u_{n}=\frac{\xi \xi_{\perp} v_{2}}{8\left(1+\xi^{2}\right)}, \\
u_{1}=\frac{\xi \xi_{\|} v_{2}}{8\left(1+\xi^{2}\right)}, \quad u_{2}=\frac{i \xi \xi_{\perp} q}{2\left(1+\xi^{2}\right)}-\frac{\xi\left(v_{2} \xi_{\|}-\xi_{\perp} \cdot v_{\|}\right)}{8\left(1+\xi^{2}\right)}, \\
\phi=\frac{\xi^{2}}{2\left(1+\xi^{2}\right)}, \quad \lambda=\frac{\xi}{2\left(1+\xi^{2}\right)} .
\end{gathered}
$$

Докажем, что все члены в $R$, пропорциональные $q$, при надлежашем выборе $E^{\prime}$ сокрашаются, и тем самым $F$ и $H$ не содержат членов, изменяюшихся на расстояниях порядка комптоновской длины волны пропорционально $1 / q$.

Из $(12),(13)$ и (17) следует, что в $R$ зависящие от $\tau_{n}$ члены, пропорциональные $q$, могут быть сгруппированы:

$$
{ }^{-} \widehat{V}^{-}\left(\tau_{n}\right)-\frac{1}{2 q}-\widehat{V}^{+}\left(\tau_{n}\right)\left[\sum_{k=0}^{\infty}\left(\frac{-1}{2 q}\right)^{k}+\widehat{V}^{+}\left(\tau_{n}\right)^{k}\right]+\widehat{V}^{-}\left(\tau_{n}\right) .
$$

Сохраняя в ${ }^{-} \widehat{V}^{-}\left(\tau_{n}\right),+\widehat{V}^{+}\left(\tau_{n}\right),-\widehat{V}^{+}\left(\tau_{n}\right)$ и $+\widehat{V}^{-}\left(\tau_{n}\right)$ члены, пропорциональные $q$, находим

$$
\begin{aligned}
-\widehat{V}^{-} & =\frac{1}{2} E^{\prime \prime}\left(\begin{array}{cc}
\sigma_{-} & 0 \\
0 & \sigma_{+}
\end{array}\right), \\
{ }^{+} \widehat{V}^{+} & =\frac{1}{2}\left(E^{\prime \prime}+2 \frac{-\xi^{2}+\xi_{\perp}^{2} / 2}{1+\xi^{2}} q\right)\left(\begin{array}{cc}
\sigma_{+} & 0 \\
0 & \sigma_{-}
\end{array}\right), \\
-\widehat{V}^{+} & =-\frac{\xi_{\perp}\left(\xi+\xi_{\|}\right)}{4\left(1+\xi^{2}\right)} q \hat{e}_{1}\left(\begin{array}{cc}
\sigma_{+} & 0 \\
0 & -\sigma_{-}
\end{array}\right), \\
+\widehat{V}^{-} & =\frac{\xi_{\perp}\left(\xi-\xi_{\|}\right)}{4\left(1+\xi^{2}\right)} q \hat{e}_{1}\left(\begin{array}{cc}
\sigma_{-} & 0 \\
0 & -\sigma_{+}
\end{array}\right),
\end{aligned}
$$

где $E^{\prime}=E^{\prime \prime}+P_{n}$.

Подставляя (21) в (20), получаем, что выражение (20) равно

$$
\frac{1}{2}\left(E^{\prime \prime}+\frac{\xi_{\perp}^{4} q^{2}}{4\left(1+\xi^{2}\right)^{2}}\left(E^{\prime \prime}+2 \frac{1+\xi_{\perp}^{2} / 2}{1+\xi^{2}} q\right)^{-1}\right)\left(\begin{array}{cc}
\sigma_{+} & 0 \\
0 & \sigma_{-}
\end{array}\right)
$$


Отсюда следует условие, определяющее $E^{\prime \prime}$ :

$$
E^{\prime \prime}\left(E^{\prime \prime}+2 \frac{1+\xi_{\perp}^{2} / 2}{1+\xi^{2}} \cdot q\right)=-\frac{\xi_{\perp}^{4} q^{2}}{4\left(1+\xi^{2}\right)^{2}}
$$

Подставляя $E^{\prime \prime}$ в $\widehat{V}(\tau)$, мы полностью определим эту величину, а следовательно и ядро интегрального уравнения (12), определяюшего эволюцию спинорных амплитуд $F$ и $H$.

Эффект нейтринных осцилляций $\nu_{\mathrm{L}} \leftrightarrow \nu_{\mathrm{R}}, \bar{\nu}_{\mathrm{R}} \leftrightarrow \bar{\nu}_{\mathrm{L}}$ массивных нейтрино в метрике Керра-Шилда с помощью полученного в данной работе $\widehat{V}$ будет исследован в дальнейшем.

Благодарности. Работа выполнена при финансовой поддержке Минобразования РФ (грант Е00-3.3-101).

\section{Список литературы}

[1] Y. Fukuda et al. Phys. Rev. Lett. 1998. V. 81. № 8. P. 1562-1567.

[2] F. Boehm et al. Phys. Rev. Lett. 2000. V. 84. № 17. P. 3764-3767.

[3] В. П. Цветков. Матем. моделирование. 1998. Т. 10. № 2. С. 103-109.

[4] В. П. Цветков. ЯФ. 2000. Т. 63. № 4. С. 765-768.

[5] Д. Е. Кумпяк, В. П. Цветков. ТМФ. 2000. Т. 125. № 2. С. 343-352.

[6] V.P. Tsvetkov, D.E. Kumpjak, V.N. Ryzukov. Spin Dynamics of a Massive Neutral Dirac Particle in the Slightly Curved Space-Time with an Arbitrary Geometry. In: Proceedings XVth Workshop on High Energy Physics and Quantum Field Theory. Eds. M. Dubinin, V. Savrin. M.: Изд. отдел УНЦ ДО, 2001. Р. 330-334. 